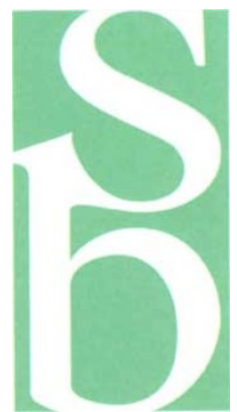

\section{The evolution of oligomerization}

What are the mechanisms that underlie the evolution of protein form? Although the details of intra- or intermolecular interactions are often moulded by gradual change involving individual residues, there is much evidence indicating that the overall architecture of a polypeptide chain is formed by the additon, duplication or deletion of entire protein domains. Recombination of such units - or exon shuffling of domain modules (reviewed in ref. 1) - is thought to have come to prominence at the time of the appearence of the first metazoans. Indeed, recombination, possibly involving entire genes, must have been central in laying down the estimated 1,000 folds now in existence ${ }^{2}$. But what of higher order structure in proteins?

How the quaternary structure of proteins has arisen during evolution presents an interesting problem. Formation of a stable, long-lived dimers or oligomers requires multiple interactions between the monomers to hold the multimeric structure togetherindeed, dimer interfaces are extensive, often $700-1500 \mathrm{~A}^{2}$ in area. How did such interactions appear during evolution? There are a number of ways in which one might imagine mutlimerization appearing. The chance interaction between two proteins could be sufficiently long lived for individual mutations to gradually reinforce the association, rendering it permanent. Alternatively, an entire dimerization domain might appear in one fell swoop; by exon shuffling, for example.

An intriguing hypothesis presented by Eisenberg and colleagues ${ }^{3}$-in their recent report of the high $(2.0 \mathrm{~A})$ resolution structure of diphtheria toxin - provides a mechanism for the appearance of protein quaternary structure that does not rely on chance association or on mutation: the nuts and bolts of the multimer interface is already built into the monomer.

\section{Domain swapping}

Diphtheria toxin (DT) is perhaps the most lethal protein on the planet; the presence of a single molecule in the cytoplasm is sufficient to kill an entire cell. The structure of DT can be described as a compact ' $Y$ ' shape, consisting of three domains. The aminoterminal $\mathrm{C}$ domain forms one arm of the $\mathrm{Y}$, the carboxy-terminal $\mathrm{R}$ domain the other arm and the $\mathrm{T}$ domain forms the stem of the $\mathrm{Y}$. In the original description of the structure, at $2.5 \AA$ resolution ${ }^{4}$, DT was observed as a dimer in the crystal, with two monomers apparently interacting with one another through an interface between their $\mathrm{R}$ domains, involving three hydrogen bonds per monomer.

Now that Eisenberg and colleagues have refined data to a higher resolution they find that a three-residue loop had been misplaced in the original structure, leading to the erroneous suggestion that this loop connected the $\mathrm{T}$ domain with the nearest $\mathrm{R}$ domain of the 'same' monomer, as perhaps might have been expected. The refined structure shows, in fact, that the loop connects the $T$ domain to the distant $\mathrm{R}$ domain of the 'other' monomer. The two $\mathrm{R}$ domains have been neatly exchanged, by rotating through $180^{\circ}$, between monomers in the dimeric DT structure. Indeed, the swap is almost perfect, with the 'shared' $\mathrm{R}$ domains making the same contacts with the $C$ domains of the partner monomers as the $\mathrm{R}$ domain does in the monomeric form of DT. However, the 'domain swap', as Eisenberg and colleagues ${ }^{3}$ call the phenomenon, does require a large rearrangement of the loop that connects the $\mathrm{T}$ domain with the 'displaced' $\mathrm{R}$ domain.

This exchange of identical domains between monomers preserves the interdomain interactions seen within the monomer, except 
that now the contacts are providing the glue of the intermolecular interaction between monomers in the dimer, effectively providing a preformed dimer interface. Oligomers could, of course, form by a similar mechanism and Eisenberg and colleagues see DT trimers, tetramers and pentamers.

Although the DT dimer does have distinct properties compared to monomeric DT-the dimers are unable to interact with cell-surface receptors-the monomers do not spontaneously form dimers (which seems to require acid $\mathrm{pH}$ ) and so it is difficult to imagine dimerization being a route for further evolution of this molecule.

\section{BS ribonuclease}

Another instance of domain swapping that does happen spontaneously, as well as conferring unique and potentially 'useful' properties on the domain-swapped dimer is seen in the case of bovine seminal ribonuclease $\mathrm{e}^{5}$ (BS RNase). BS RNase is unusual in that it is the only dimeric RNases so far characterized. Furthermore, the dimers exist in two different quaternary conformations: in one form the monomers are linked by two inter-subunit disulphide bonds; in the other form there are no cystine bridges linking the monomers, rather, identical amino-terminal segments have been swapped between monomers, so that the forces holding the two monomers together are purely non-covalent. As in the DT dimer, the swapped amino-terminal segments interact with their partner monomers in precisely the same fashion as that seen in the non-swapped covalent dimer, the only difference between the two structures (other than the disulphide bridges) being the conformation of the hinge about which the animo-terminal segments rotate. The domain swapped dimer, unlike the covalent dimer, is endowed with allosteric, regulatory properties that may well confer some small advantage to the cow and thus be a substrate for natural selection.

Eisenberg and colleagues ${ }^{3}$ further expect that the domain swopped dimer, initially held together by what is the equivalent of the intradomain interface in the isolated monomer, will be stabilized by mutations at a secondary domain interface, which will further favour dimerisation, and hence the 'advantageous' properties of the dimer. Eventually, a situation could arise where the monomer, having accumulated numerous mutations that promote oligomerization, would no longer be stable with respect to the oligomeric form.

The beauty of this hypothesis is in the 'preevolved' nature of the dimer interface. There is no need to imagine individual mutations gradually facilitating the transition from monomer to dimer-just the ability of monomers to exchange identical domains with their partners. The new dimer interactions would have been long established and 'refined' by natural selection in the context of the interdomain interface in the monomer.

What of the evidence that such a mechanism operated during evolution? Eisenberg and colleagues note that oligomeric interfaces have been shown to have the same structural characteristics as monomer interiors ${ }^{5}$, an observation at least consistent with the hypothesis. Furthermore, a number of intertwined protein oligomers may have evolved by this route: for example, IL-5 has a dimer interface similar to an interdomain interface in GM-CSF and may have been derived from the latter by domain swopping. Unlike the initial step of the mechanism itself, accumulation of evidence either for or against the hypothesis is likely to be a slow and gradual process.

1. Patthy, L. Curr. opin. Struct. Biol 4, 383-392 (1994).

2. Chothia, C. Nature 357, 543-544 (1992).

3. Bennet, M.J., Choe, S. \& Eisenberg,

D. Proc. natn. Acad. Sci. U.S.A. 91, 3127-3131 (1994).

4. Choe, S. et al. Nature 357, 216-222

(1992).

5. Miller, S. Protein Engng 3, 77-83 (1989).

6. Piccoli, R. et al. Proc. natn. Acad. Sci. U.S.A. 89, 1870-1874 (1992).

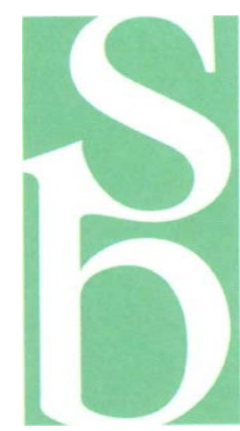

